\title{
Potentiometric enzyme channeling immunosensor for proteins
}

\author{
Daniel V. Brown* \& Mark E. Meyerhoff* \\ Department of Chemistry, The University of Michigan, Ann Arbor, MI 48109-1055, USA \\ (Received 10 August 1990; revised version received 5 November 1990; accepted 6 November 1990)
}

\begin{abstract}
A potentiometric immunosensor for the detection of human IgG has been developed using an asymmetric, ion-selective membrane with immobilized adenosine deaminase and IgG. A protein A-alkaline phosphatase conjugate binds to the immobilized IgG, creating a bienzymatic catalytic layer. In the presence of sample IgG, the conjugate does not bind to the membrane. Instead, the intermediate in the two-step reaction (adenosine) must diffuse to the membrane surface, reducing the rate of product (ammonium) formation within the diffusion layer detected by the membrane. The immunosensor demonstrated is for the determination of IgG. A simplified model is described to predict the maximum rate enhancement for the 'channeled' versus 'unchanneled' reaction mechanisms.
\end{abstract}

Keywords: immunosensor, pseudo-homogeneous, enzyme channeling, immunoglobulin $\mathrm{G}$, ion-selective electrode.

\section{INTRODUCTION}

In the early 1980 s the concept of an 'enzyme channeling immunoassay' (ECIA) was introduced by Litman et al. (1980). This type of assay is conducted in a pseudo-homogeneous format. The format is particularly useful in the event that an enzyme conjugate of a large antigen (e.g. a protein) is not inhibited by antibody or binding protein association. In fact, it is preferred that this not be the case. Thus, the technique is suitable for the quantitation of large analyte molecules without the need for multiple washing and incubation steps associated with more conventional heterogeneous enzyme immunoassay procedures.

*Present address: Baxter Healthcare Corporation, Miami, FL 33172, USA.

₹To whom all correspondence should be addressed.
The channeling strategy involves the use of two or more enzymes related by the fact that the product of one enzyme is the substrate for another. The interaction between an antigen and antibody brings one of the enzymes in close proximity to the second. The product of the first enzymatic reaction is then said to be 'channeled' into the second reaction. The basis for signal modulation is that a binding interaction facilitates the rapid conversion of the initial substrate into final product. In the absence of that binding interaction, the product of the first enzyme in the catalytic sequence must diffuse a greater distance to the second enzyme, a process which then becomes the rate-limiting step in the process. Many catabolic and anabolic processes in living tissues involve multi-enzyme complexes in order to circumvent this limitation. Fig. 1 depicts this scenario.

Historically, ECIAs have been developed by 


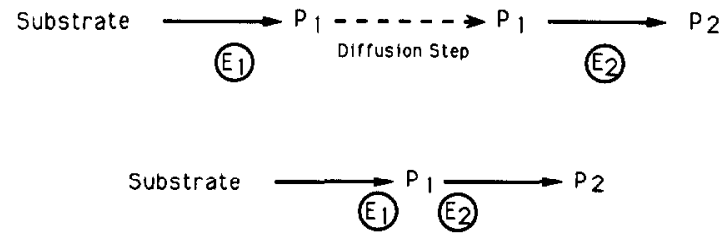

Fig. 1. Two-enzyme catalytic sequence. Top: 'unchanneled' reaction. Bottom: 'channeled' reaction.

immobilizing one enzyme on a solid support along with an antibody or analyte. Immunological reactions at the solid phase surface then cause the second enzyme in the bulk solution to partition into a catalytically active layer at the boundary of the solid phase. To date, cellulose paper and agarose have been used as solid supports for ECIA.

The original work of Litman et al. (1980) was followed by several similar assays (Litman et al., 1983; Ullman et al., 1983; Chen et al., 1984; Terouanne et al., 1986a, b; Carrie et al., 1986). Although the primary advantage of the ECIA format is that it permits pseudo-homogeneous assay conditions, two of the reports (Litman et al., 1983; Chen et al., 1984) are not, in practice, homogeneous assays. Instead they are conducted by incubating an immobilized bioreagent strip in sample/conjugate solution, then transferring the strip 'without washing' to a separate substrate solution. In addition, most of the ECIA systems reported to date require lengthy substrate incubation times to obtain enough color or luminescence from the product of the two enzymes within the reaction layer adjacent to the solid phase.

The goal of the research described in this report was to develop an enzyme channeling immunoassay system with electrochemical detection. Such an assay would have the following potential advantages: no interferences from turbid or colored sample matrices, inexpensive instrumentation, and a short assay time. The last advantage would be because electrochemical detectors sense the concentrations of species in the diffusion layer adjacent to the device and not throughout the entire sample.

In the model system described herein, a potentiometric ammonium-selective membrane, with a covalently immobilized enzyme and immunoglobulin, is employed. The ammoniumselective membrane and the immobilization technology is nearly identical to that described in previous work (Cha \& Meyerhoff, 1989). The enzymes utilized and reactions they catalyze are as follows:

$$
\begin{gathered}
\text { 5'-AMP } \underset{\mathrm{ALP}}{\longrightarrow} \text { Adenosine + Phosphate } \\
\text { Adenosine } \underset{\mathrm{ADA}}{\longrightarrow} \text { Inosine + Ammonia }
\end{gathered}
$$

Alkaline phosphatase (ALP) and adenosine deaminase (ADA) sequentially facilitate the production of ammonium ions from adenosine monophosphate (AMP). Figure 2 illustrates the final assay format incorporating a membrane with immobilized ADA and human immunoglobulin G (hIgG). A protein A-ALP conjugate binds to the membrane-immobilized hIgG. Protein A is a $42 \mathrm{kDa}$ polypeptide isolated from Staphylococcus aureus which binds to the IgG1, IgG2, and IgG4 subclasses through an interaction with the $F c$ region of the immunoglobulin (Kronvall \& Williams, 1969). It is bivalent, and also binds human IgM and IgA (Goudswaard et al., 1978). The conjugate must compete, however, with sample hIgG, if present. Sample hIgG reduces the amount of conjugate bound to the membrane surface, increasing the time required for adenosine to diffuse to ADA, diminishing the signal arising from the formation of ammonium ions at the surface of the membrane electrode detector.

\section{EXPERIMENTAL}

\section{Reagents}

Adenosine deaminase (EC 3.5.4.4) from calf intestine, alkaline phosphatase (EC 3.1.3.1) from calf intestine, adenosine, adenosine 5 '-monophosphate (AMP), tris buffer (tris[hydroxymethyl] amino-methane), 25\% glutaraldehyde (aqueous), bis-tris propane (1,3-bis[tris \{hydroxymethyl\} methylamino]propane). CAPS (3-[cyclohexylamino]-1-propane-sulfonic acid), and agarose were obtained from Sigma Chemical (St. Louis, MO, USA). Nonactin, cellulose triacetate, and 1,1'-carbonyldiimidazole (CDI) were provided by Fluka US (Ronkonkoma, NY). Controlled pore glass was the product of Dow Diagnostics Division (Indianapolis, IN, USA). All other chemicals were of reagent grade. Deionized, 16.6 $\mathrm{M} \Omega$ water was used to prepare all aqueous solutions. 


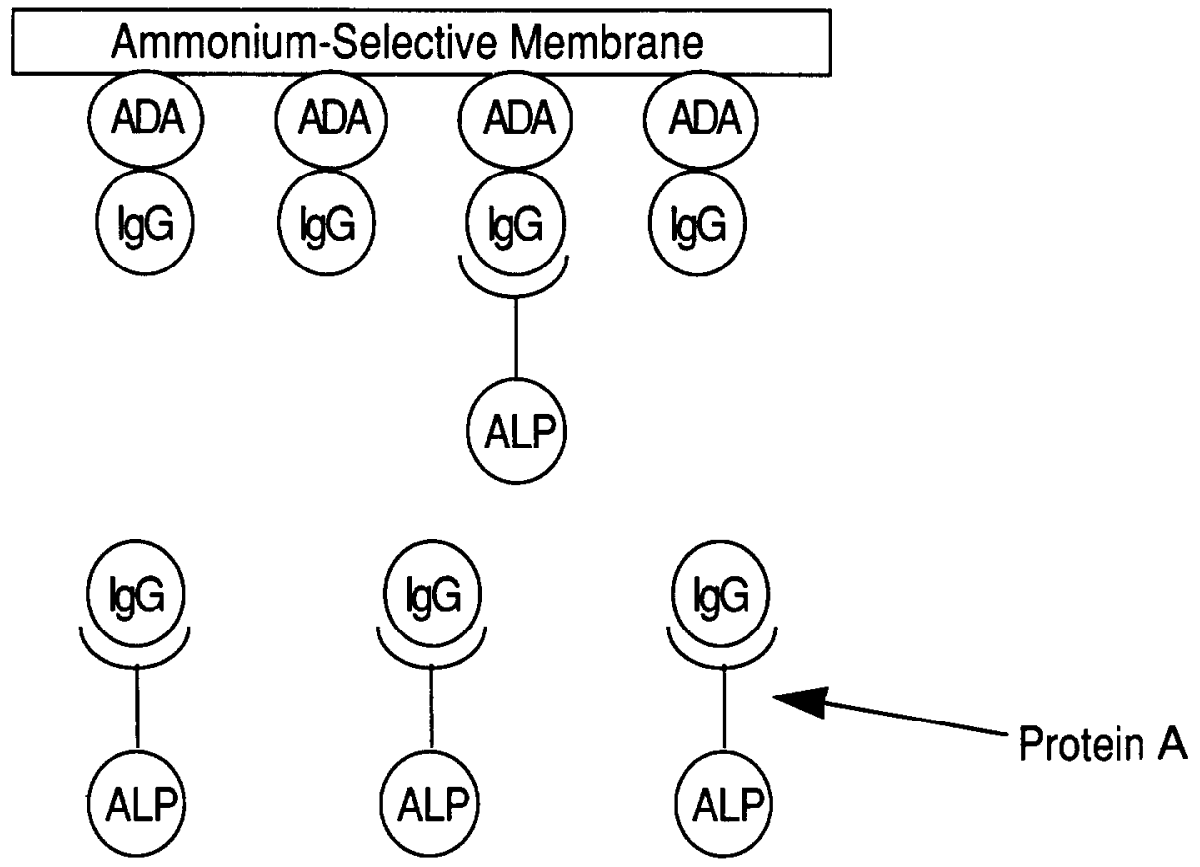

Fig. 2. Enzyme channeling immunosensor membrane with immobilized ADA and hIgG in conjunction with a protein A-ALP conjugate.

\begin{abstract}
Apparatus
Potentiometric data were recorded in two fashions. Multichannel experiments were performed using a high impedance amplifier connected to a Data Translations 2801 analog-todigital converter board installed in a Zenith Z-150 series microcomputer. Sampling rates of 1 to $0 \cdot 1 \mathrm{~Hz}$ were selected with Data Translations Labtech Notebook software. For single channel measurements, the signal from an Accumet model $825 \mathrm{MP}$ pH/mV meter (Fisher Scientific) was recorded by a Heath model SR-204 stripchart recorder. ISE membranes were mounted in Phillips ISE-561 electrode bodies (Glasblaserei Moller, Zurich). A saturated calomel electrode (Fisher) was used as a reference. A Fisher model 80 thermostat provided controlled temperature circulating water for experiments performed at $310 \mathrm{~K}$.
\end{abstract}

\section{Preparation of immunosensor membrane}

An asymmetric, cellulose triacetate, ammoniumselective membrane was cast similar to the previously-reported method (Cha \& Meyerhoff, 1989). The immobilization of ADA was carried out via the CDI method. This involved immersing the hydrolyzed side of the membrane (containing the hydroxyl groups) into $20 \mathrm{ml}$ of cold $(277 \mathrm{~K})$ distilled water and adding excess CDI (about $50 \mathrm{mg}$ ) in 10-15 mg portions over a $15 \mathrm{~min}$ period. After a brief washing with cold water, $15 \mu \mathrm{l}$ of a solution containing $200-400 \mu \mathrm{g}$ of ADA was applied to the activated surface of the membrane. The membrane was then soaked in tris- $\mathrm{HCl}$ assay buffer in order to block any remaining active groups, and rinsed with $0.1 \mathrm{M}$ glycine buffer, $\mathrm{pH} 2 \cdot 2$, briefly (approximately $30 \mathrm{~s}$ ) to remove any nonspecifically adsorbed enzyme. A solution of $2.5 \%$ glutaraldehyde in $0.1 \mathrm{M}$ phosphate buffer,

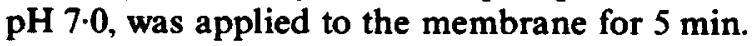
After rinsing with water, $\mathrm{hIgG}(200-800 \mu \mathrm{g}$ in $15 \mu \mathrm{l}$ phosphate buffer) was applied to the membrane for at least $4 \mathrm{~h}$ at $277 \mathrm{~K}$. This immobilization sequence essentially produced a microscopically laminated bioelectrode consisting of the base asymmetric membrane, an enzyme monolayer, and an immunoglobulin monolayer. Before use in assays or other studies, the membranes were rinsed in $0.1 \mathrm{M}$ guanidine- $\mathrm{HCl}$, pH 3.0, $10 \mathrm{wt} \%$ glycerol, for $1 \mathrm{~min}$ to remove any nonspecifically adsorbed proteins.

\section{Homogeneous immunosensor assay protocol}

For most of the studies reported, the following assay protocol was utilized. A circulating water- 
jacketed beaker $(310 \mathrm{~K})$ provided a temperaturecontrolled environment. First, the sample mixtures containing hIgG $(200 \mu \mathrm{l})$ were preincubated for $5 \mathrm{~min}$ with protein-A-ALP conjugate $(10 \mu \mathrm{l})$. The sensor was then immersed in this solution ( $5 \mathrm{ml}$ total volume) for an additional $10 \mathrm{~min}$. One milliliter of substrate solution (AMP in assay buffer), such that the final substrate concentration was $1 \mathrm{mM}$, was stirred into the assay buffer for approximately $5 \mathrm{~s}$. With no further stirring, the potential of the biosensor versus the reference electrode was recorded as a function of time. The potential change over the first minute of response correlates to the rate of product formation in the immobilized reaction layer. After this measurement, the sensor was regenerated by washing for $1 \mathrm{~min}$ in a $0.1 \mathrm{M}$

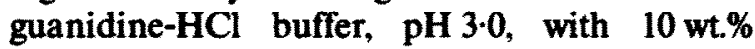
glycerol. The assay buffer consisted of $0.1 \mathrm{M}$ tris$\mathrm{HCl}, \mathrm{pH} 8.5$, with $10 \mathrm{wt} \%$ glycerol. The glycerol served to reduce non-specific adsorption of the protein-A-ALP conjugate and increase the viscosity of the buffer (i.e. decrease diffusion).

\section{Correlation study}

Nine samples of human serum were obtained from the Clinical Pathology Laboratory of the University of Michigan Hospital. These were selected randomly and had been analyzed by rate nephelometry for IgG on a Beckman Array instrument using reagents and protocol recommended by the manufacturer. In order to remove endogenous sodium and potassium, both interferents for the membrane electrode, each sample was passed through $2 \mathrm{~m}$ of Nafion (id $0.625 \mathrm{~mm}$ ) tubing immersed in $2 \mathrm{M} \mathrm{MgCl}_{2}$. Magnesium ion is divalent, and produces a response 1000 times less than sodium in the nonactin-based ISE. After this clean-up procedure, samples were diluted 50-fold with assay buffer. Each sensor was calibrated, then a sample was tested. If the sensor response was outside the calibration range, the sample was analyzed again at a different dilution, depending upon the initial result. Average IgG values for two determinations were compared to those obtained by rate nephelometry.

\section{RESULTS AND DISCUSSION}

The development of this immunosensor device based on the principles of enzyme channeling is complicated by the fact that four large proteins are involved, each requiring specific conditions for optimal biological activity (e.g. ionic strength, temperature and $\mathrm{pH}$ ). For example, alkaline phosphatase, as the name implies, has maximum activity at a pH of 10 (McComb \& Bowers, 1972). ADA has maximum activity in the range $6<\mathrm{pH}<8$ (Orsi et al., 1972). In preliminary studies, both immobilized and free ADA were observed to have maximum activity at approximately $\mathrm{pH} \mathrm{8.8,} \mathrm{as} \mathrm{measured} \mathrm{with} \mathrm{an}$ ammonium-selective electrode. The difference in the maximum activity obtained in this study and that obtained in the cited work may be attributed to the different buffer systems used. In addition, it is known that maximum binding of protein $\mathbf{A}$ with IgG occurs near neutral pH (Delacroix \& Vaerman, 1979). Therefore, the final assay buffer pH (8.5) reflects a compromise condition such that protein A binding can still occur, while the signal generating enzymes (ALP and ADA) exhibit near optimal activity.

To select an appropriate assay binding time, an aliquot of protein-A-ALP conjugate $(10 \mu l)$ was incubated with an immunosensor, then the sensor was rinsed with assay buffer and immersed in fresh buffer solution. Upon the addition of substrate (AMP), the signal generated by the two-step reaction was recorded. This procedure was repeated using variable incubation times. Between each assay, the sensor was washed with regeneration buffer $(0 \cdot 1 \mathrm{M}$ guanidine- $\mathrm{HCl}, \mathrm{pH} 3 \cdot 0,10 \%$ glycerol). As Fig. 3

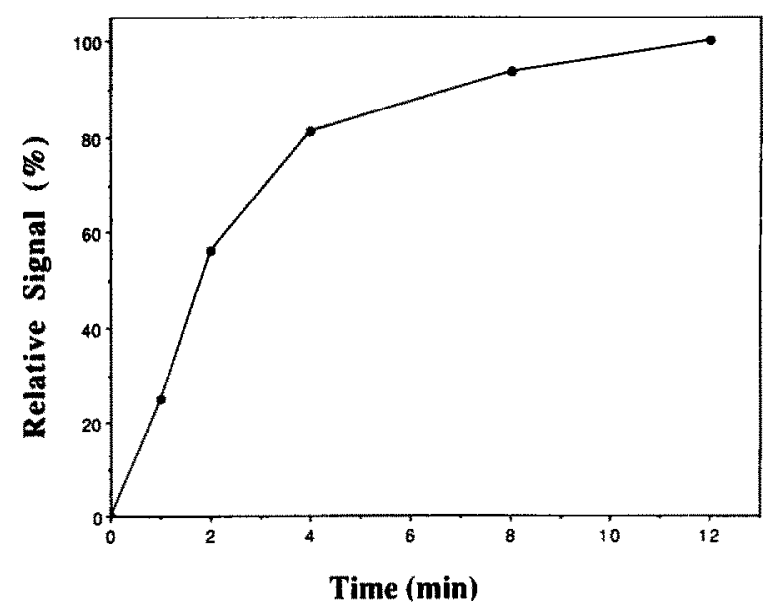

Fig. 3. Time-dependent binding of pmotein-A-ALP conjugate to immunosensor membrane (membrane prepared with $600 \mu \mathrm{g}$ immobilized $\mathrm{hIg}$, assay performed with $10 \mu$ l conjugate). 


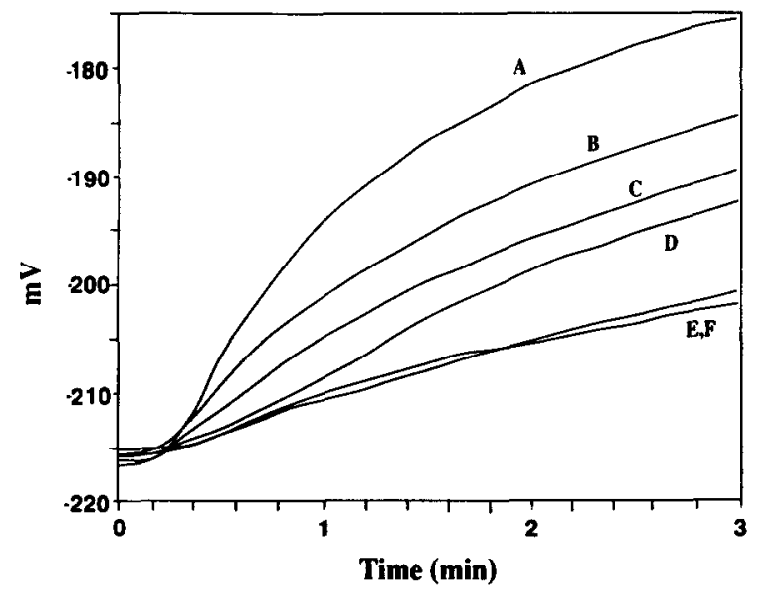

Fig. 4. Enzyme channeling immunosensor response curves for several analyte concentrations. (A) $0 \mu \mathrm{g} \mathrm{ml^{-1 }} h \mathrm{IgG},(B)$

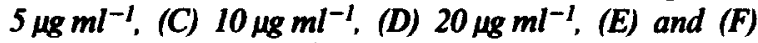
$50 \mu \mathrm{g} \mathrm{ml} \mathrm{l}^{-1}$ and blank response.

illustrates, more than $80 \%$ of the total conjugate binding occurs within $5 \mathrm{~min}$. In subsequent, competitive binding assays for hIgG, an incubation time of $10 \mathrm{~min}$ was employed to ensure near-maximum binding of the conjugate to the surface of the sensor.

Following the competitive binding assay protocol given in the Experimental section, the responses shown in Fig. 4 were recorded for several different concentrations of analyte hIgG. It is notable that the blank signal, that arising from the addition of AMP substrate in the absence of the protein-A-ALP conjugate, is equivalent to the signal for the assay containing $50 \mu \mathrm{g} \mathrm{ml}^{-1}$ hIgG. This phenomenon was observed repeatedly with sensors constructed with various amounts of $h I g G$ in the immobilized layer, and is due in part to trace levels of ALP in the immobilized ADA, some adenosine impurity in the AMP substrate, and electrode drift in the unstirred solution. Thus, in the presence of high levels of sample hIgG, the so-called 'unchanneled' rate of the two-step reaction is dictated by the background blank signal.

The rates of product formation were determined by performing linear regressions of the initial rate data. This was facilitated by the digital recording of potentials. Such a reduction of the Fig. 4 data is shown in Fig. 5 (see inset graph). Initially, the addition of sample hIgG causes a dramatic reduction in two-step reaction rate. When greater than $5 \mu \mathrm{g} \mathrm{ml}^{-1} \mathrm{hIgG}$ was present in the assay solution, the dose-response

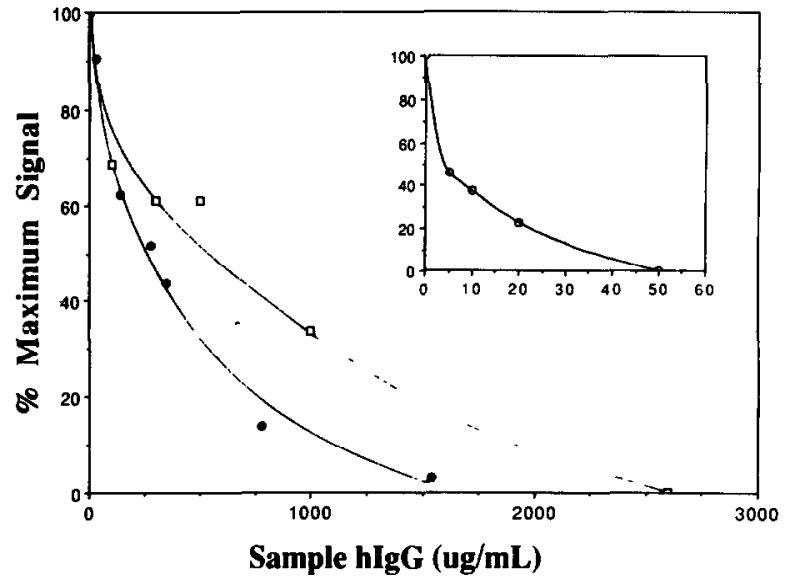

Fig. 5. Family of calibration curves for immunosensors with varying amounts of immobilized hIgG: O, $500 \mu \mathrm{g}$; ㅁ $800 \mu \mathrm{g}$. Inset: $200 \mu \mathrm{g}$ immobilized hIgG. Data points are an average of two determinations with given sensor at each hIgG concentration.

curve was fairly linear until reaching zero (relative to blank).

As one would expect in a competitive assay arrangement, the amount of immobilized hIgG proved to affect the detection limits. Because a given amount of protein-A-ALP conjugate is in equilibrium between free $\mathrm{hIgG}$ and immobilized hIgG, an increase in the amount of immobilized hIgG will cause the equilibrium distribution of conjugate molecules to favor the membranelinked hIgG. Several membranes were prepared with identical amounts of ADA, but with variable amounts of hIgG. All were tested with the same aliquot of conjugate $(10 \mu \mathrm{l})$. The calibration curves for three different membranes are shown in Fig. 5. As can be seen, a significant enhancement in detection capabilities is observed when the amount of immobilized hIgG is reduced.

One shortfall of this immunosensor's design is durability. Indeed, repeated regeneration of the sensor with $0.1 \mathrm{M}$ guanidine- $\mathrm{HCl}$ buffer, $\mathrm{pH} \mathrm{3.0,}$ is possible, but only up to a point. After 8-10 cycles, a considerable decrease in the binding ability of protein A to the immobilized hIgG is observed. Little loss in immobilized ADA activity occurs, even when the sensor is recycled 20 times. Thus, reuse of the sensor appears to be limited by the gradual denaturation of the immobilized hIgG upon exposure to the regeneration buffer. Perhaps the use of a milder regeneration agent than the $\mathrm{pH} 3.0$ guanidine- $\mathrm{HCl}$ buffer would extend the lifetime of the proposed sensor. 
The inability to reuse the proposed enzyme channeling sensor repeatedly precludes a typical study of the precision attainable with this device. For this reason, a measure of precision was made in a different fashion. Two different sensors were prepared in the manner previously described, and then used to measure two different sample concentrations of hIgG several times. The first was an assay of the variability of the zero-dose signal. One sensor repeated this measurement five times and yielded a rate of $13.8 \pm 0.7 \mathrm{mV} \mathrm{min}^{-1}$ at the $95 \%$ confidence level. The second sensor, assaying a $20 \mu \mathrm{g} \mathrm{ml}^{-1} \mathrm{hIgG}$ standard $(n=5)$ yielded a value of $4.1 \pm 0.6 \mathrm{mV} \mathrm{min}^{-1}$. The latter signal represents $29 \%$ of the maximum signal at zero analyte level.

The enzyme channeling immunosensor was compared with the rate nephelometry assay method for the determination of hIgG in nine human serum samples. Samples were provided by the Clinical Pathology Laboratory of the University of Michigan Hospital. Results from the two methods are compared in Fig. 6, and indicate that the immunosensor method compares favorably with the nephelometric method.

The enzyme channeling immunosensor exhibits large increases in the 'channeled' rate versus the 'unchanneled' rate. In fact, at maximum levels of analyte $\mathrm{hIgG}$ concentrations, the assay signal is equal to the blank signal. As stated previously, the blank signal arises from electrode drift (the reproducible positive

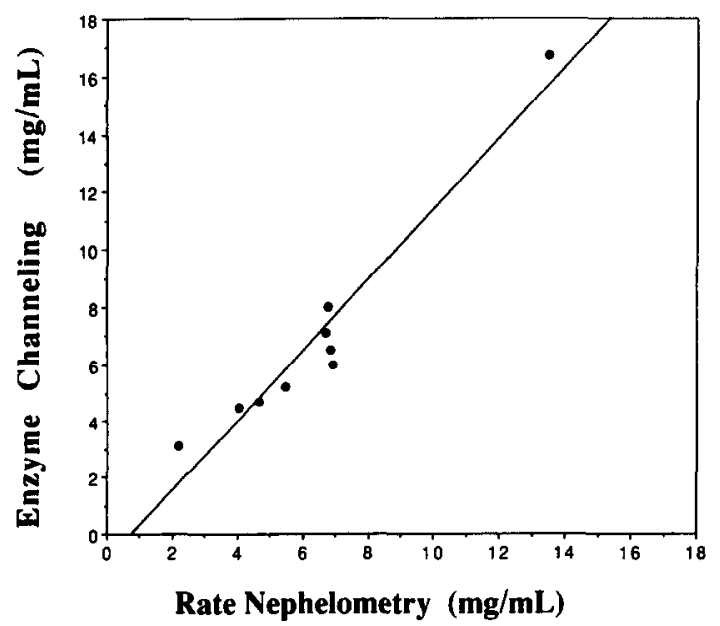

Fig. 6. Correlation of enzyme channeling immunosensors with rate nephelometry method for serum hIgG. $(\mathrm{y}=1.23 \mathrm{x}-0.941, \mathrm{R}=0.970)$.

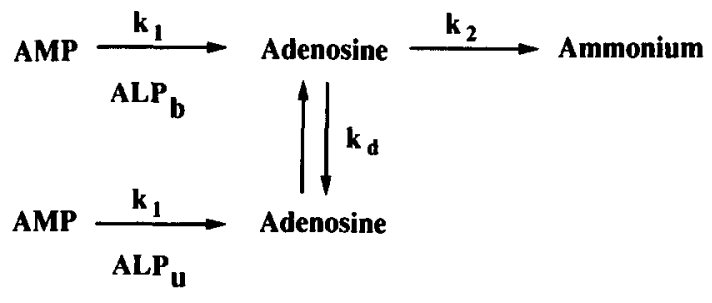

Fig. 7. Reaction scheme for enzyme channeling immunosensor. Subscripts $b$ and $u$ indicate bound and unbound species, respectively.

potential change due to absence of stirring), ALP impurity in the immobilized ADA, and adenosine impurity in $5^{\prime}$-AMP.

Figure 7 outlines the simplified pathways contributing to the production of a signal from the sensor when protein A-ALP is present in the assay mixture. The primary factor contributing to the background signal (in the presence of protein-A-ALP conjugate) is ammonium produced from adenosine diffusing into the unstirred layer adjacent to the electrode membrane. This could be referred to as the 'unchanneled signal'. It can be assumed that the rate of diffusion of protein-A-ALP conjugate from the bulk solution into the unstirred layer is very small compared with that of adenosine.

Ullman et al. (1980) have suggested a value known as the 'substrate capture efficiency', $S$ (see eqn. 1), calculated from the reaction rate constants $\left(k_{1}\right.$ and $\left.k_{2}\right)$ and diffusion coefficient $\left(k_{\mathrm{d}}\right)$ shown diagrammatically in Fig. 7.

$$
S=\frac{k_{2}}{k_{\mathrm{d}}+k_{2}}
$$

To increase $S, k_{\mathrm{d}}$ must be minimized and $k_{2}$ maximized. As $S$ increases, the detection limit is lowered because the background signal decreases and the zero-dose signal is enhanced. The diffusion coefficient of adenosine itself may be manipulated by increasing the viscosity of the buffer solution. In the present study, glycerol was added to the buffer, which has the two-fold effect of reducing non-specific adsorption of proteins and increasing buffer viscosity. The pseudo-firstorder rate constant $k_{2}$, may be maximized by favoring the $\mathrm{pH}$ maximum of immobilized ADA or by loading the membrane with as high an amount of ADA as possible. Ideally, the overall two-step reaction rate is dependent upon the amount of conjugate bound to the membrane 
surface, not the amount and/or activity of immobilized ADA.

Considering diffusion processes, a better understanding of the advantage obtained by not stirring the assay buffer after the addition of substrate can be gained. The root-mean-square displacement of a particular molecule at time $t$ can be approximated by $(2 D t)^{1 / 2}$, where $D$ is the diffusion coefficient for the molecule and $t$ is time. Typical values of $D$ for small organic molecules in aqueous solutions are approximately $5 \times 10^{-6} \mathrm{~cm}^{2} \mathrm{~s}^{-1}$, and 100 times smaller for globular proteins (McClintock \& Purdy, 1987). Assuming that diffusion of adenosine occurs linearly from a planar surface with bound conjugate, a 'diffusion layer' grows in thickness with time so that in $10 \mathrm{~s}$, a layer $0.1 \mathrm{~mm}$ thick is formed. In $1000 \mathrm{~s}$, the layer is $1.0 \mathrm{~mm}$ in thickness. Using the larger value to represent the thickness of the diffusion layer at the end of the measurement sequence of the enzyme channeling immunosensor, further calculations of product formation were made. Implicit in this is the assumption that the diffusion coefficient of adenosine is similar to that of glucose, $6.73 \times 10^{-6} \mathrm{~cm}^{2} \mathrm{~s}^{-1}$ (Weast, 1981). After $1000 \mathrm{~s}$, a roughly hemispherical volume of approximately $30 \mu 1$ (see Fig. 8(a)) would contain all the adenosine produced by bound protein-A-ALP that could conceivably be converted to ammonium and detected by the electrode. The diffusion layer is approximately hemispherical because the radius of the membrane is on the order of the layer thickness.

In the extreme case of maximum 'channeling', all of the protein-A-ALP aliquot will be specifically bound to membrane-immobilized hIgG within the diffusion layer. This is illustrated in Fig. 8(a). At the opposite extreme, no proteinA-ALP conjugate will be specifically bound nor nonspecifically adsorbed to the membrane (see Fig. 8(b)). However, a portion of the conjugate will still be present within the diffusion layer due to the dilution of the conjugate throughout the entire assay volume. These unbound molecules of protein-A-ALP conjugate can catalyze AMP to adenosine, resulting in a background 'unchanneled' signal.

To approximate the maximum 'channeled'/ 'unchanneled' ratio, one need only consider the relative ratio of volume elements in which the adenosine is liberated from ALP. In the present system, the final assay volume was $6.0 \mathrm{ml}$. Thus

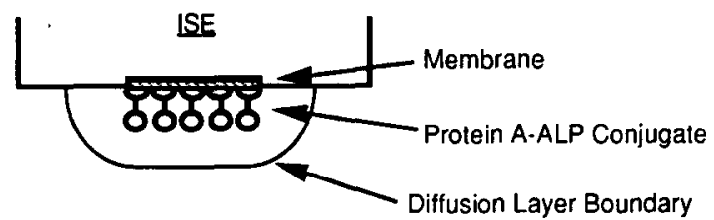

(a)

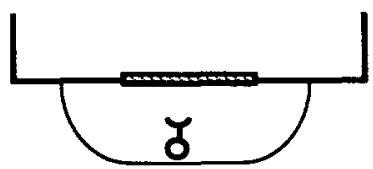

$\zeta$

(b)
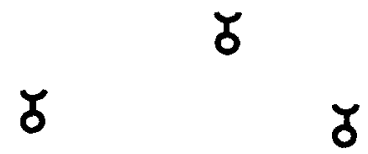

Fig. 8. Scenarios for (a) maximum 'channeled' and (b) maximum 'unchanneled' pathway reaction rates.

an enhancement factor of approximately 200 -fold is expected. Experimentally, rate enhancement factors of approximately 20-fold are observed when the blank signal is subtracted from the response at zero dose ('channeled') and high hIgG concentration ('unchanneled'). More accurate measurement of the empirical rate enhancement is difficult because of the high background signal. In addition, the theoretical model is somewhat limited in that it assumes that the solution is totally quiescent, whereas in reality, the stirrer was turned off when substrate was added. The model also does not account for nonspecific adsorption of conjugate.

In summary, a novel biosensor based upon the concept of enzyme channeling has been described. The sensor offers the advantages of a pseudo-homogeneous assay protocol and inexpensive instrumentation. The ability of the sensor to achieve rate enhancements has been proved both theoretically and empirically. While the selection of enzyme labels and the detector device utilized here may not be practical for routine measurements, it seems likely that the concept described here could be adapted to a number of other electrochemical detectors (and enzyme labels) to design immunosensors, including disposable devices, with more practical analytical utility. The ability of the sensor to perform as such in a pseudo-homogeneous mode is due to the fact that the membrane senses the activity of product ions in the unstirred layer 
adjacent to the membrane, as opposed to many optical devices which measure the signal from an integrated small volume or area at the surface of a solid phase. The latter requires much higher concentrations of product be formed before observing a signal above the background.

\section{ACKNOWLEDGMENTS}

We thank the National Science Foundation (Grant \#8813952) for financial support of these studies, and Ms Sharon Bordine of the Clinical Pathology Laboratory, University of Michigan Hospitals for providing human serum samples.

\section{REFERENCES}

Carrie, M., Terouanne, B., Brochu, M., Nicolas, J. \& dePaulet, A. C. (1986). Bioluminescent immunoassays of progesterone: a comparative study of three different procedures. Anal. Biochem., $154,126$.

Cha, G. S. \& Meyerhoff, M. E. (1989). Potentiometric ion- and bio-selective electrodes based on asymmetric cellulose acetate membranes. Talanta, 36, 271.

Chen, R, Weng, L., Sizto, N. C., Osorio, B., Hsu, C., Rodgers, R. \& Litman, D. J. (1984). Ultrasoundaccelerated immunoassay, as exemplified by enzyme immunoassay of choriogonaditropin. Clin. Chem., 30, 1446.

Delacroix, D. \& Vaerman, J. P. (1979). Simple purification of goat $\operatorname{IgG}_{1}$ and $\operatorname{IgG}_{2}$ subclasses by chromatography on protein A-Sepharose at various pH. Mol. Immunol., 16, 837.

Goudswaard, J., van der Donk, J. A, Noordzij, A, van Dam, R. H. \& Vaerman, J. P. (1978). Protein A reactivity of various mammalian immunoglobulins. Scand. J. Immunol., 8, 21.

Kronvall, G. \& Williams, R C. (1969). Differences in anti-protein $A$ activity among $\operatorname{IgG}$ subgroups. J. Immunol., 103, 828.

Litman, D. J., Hanlon, T. M. \& Ullman, E. F. (1980). Enzyme channeling immunoassay: a new homogeneous enzyme immunoassay technique. Anal. Biochem., 106, 223.

Litman, D. J., Lee, R. H., Jeong, H. J., Tom, H. K, Stitso, S. N., Sizto, N. C. \& Ullman, E. F. (1983). An internally referenced test strip immunoassay for morphine. Clin. Chem., 29, 1598.

McClintock, S. A. \& Purdy, W. C. (1987). Homogeneous voltammetric immunoassay of haptens. In Electrochemical Sensors in Immunological Analysis, ed. T. T. Ngo, Plenum, New York, pp. 77-86.

McComb, R. B. \& Bowers, G. N. (1972). Study of optimum buffer conditions for measuring alkaline phosphatase activity in human serum. Clin. Chem., $18,97$.

Terouanne, B., Carrie, M., Nicolas, J. \& dePaulet. A. C. (1986a). Bioluminescent immunosorbent for rapid immunoassays. Anal. Biochem., 154, 118.

Terouanne, B., Nicolas, J. \& dePaulet, A. C. (1986b). Bioluminescent assay for $a$-fetoprotein. Anal. Biochem., 154, 132.

Ullman, E. F., Gibbons, I., Litman, D., Weng, L. \& DiNello, R. (1983). Highly sensitive enzyme channeling immunoassay for macromolecules. Dev. Immunol., 18, 247.

Weast, R. C. (1981). CRC Handbook of Chemistry and Physics, 62nd edn, CRC Press, Boca Raton, FL. 